\title{
Is the Coefficient of Eddy Potential Vorticity Diffusion Positive? Part I: Barotropic Zonal Channel
}

\author{
V. O. IVCHENKO ${ }^{a}$ \\ University of Southampton, and National Oceanography Centre, Southampton, United Kingdom \\ V. B. ZALESNY \\ Institute of Numerical Mathematics, Moscow, Russia \\ B. SINHA \\ National Oceanography Centre, Southampton, United Kingdom
}

(Manuscript received 9 November 2017, in final form 8 May 2018)

\begin{abstract}
The question of whether the coefficient of diffusivity of potential vorticity by mesoscale eddies is positive is studied for a zonally reentrant barotropic channel using the quasigeostrophic approach. The topography is limited to the first mode in the meridional direction but is unlimited in the zonal direction. We derive an analytic solution for the stationary (time independent) solution. New terms associated with parameterized eddy fluxes of potential vorticity appear both in the equations for the mean zonal momentum balance and in the kinetic energy balance. These terms are linked with the topographic form stress exerted by parameterized eddies. It is demonstrated that in regimes with zonal flow (analogous to the Antarctic Circumpolar Current), the coefficient of eddy potential vorticity diffusivity must be positive.
\end{abstract}

\section{Introduction}

Mesoscale eddies are a very important element of the global ocean since they usually account for the main peak in the kinetic energy spectrum (Kamenkovich et al. 1986; McWilliams 2008; Wunsch and Stammer 1995). This means that ocean models have to either resolve or parameterize them. To resolve the mesoscale, horizontal grids in models must be much smaller than the internal Rossby radii of deformation. Improvements in computing capability (both memory and speed) allow us to run global models with high resolution. However, weak stratification in the polar regions and the associated small internal Rossby radii $(2-3 \mathrm{~km})$ still preclude adequate resolution to explicitly resolve eddies in these areas. Another significant problem is the appearance of strong internal variability with increasing resolution. Small disturbances can result in

\footnotetext{
${ }^{a}$ Retired.
}

Corresponding author: Vladimir Ivchenko, voi@noc.soton.ac.uk energetic noise, which can only be removed by averaging over ensembles of numerical experiments; for example, the Met Office routinely runs ensembles of 10 members for decadal predictions and 42 members for seasonal prediction (Smith et al. 2007). Rather than employing an ensemble of high-resolution model simulations to realistically represent eddies and their effects on the mean flow, another approach is to utilize lower-resolution models and include a parameterization of the important effects of the eddies on the large-scale circulation. It is very likely that mesoscale eddy parameterization "will be needed for some decades into the future" (Bachman and Fox-Kemper 2013).

Parameterization of mesoscale eddies is important not only for practical reasons (reduced computational expense) but also for theoretical reasons: a physically correct parameterization allows us to better understand the dynamics of eddy-eddy and eddy-mean flow interactions, that is, fundamental parts of geophysical fluid dynamics. There have been many studies devoted to this problem (e.g., Green 1970; Welander 1973; Marshall 
1981; Ivchenko 1984; Gent and McWilliams 1990; Ivchenko et al. 1997; Killworth 1997; Treguier et al. 1997; Olbers et al. 2000; Wardle and Marshall 2000; Olbers 2005; Eden 2010; Marshall and Adcroft 2010; Ringler and Gent 2011; Marshall et al. 2012; Ivchenko et al. 2013, 2014a,b; and many others).

The most popular approach to parameterization is use of the so-called diffusive parameterization, that is, proportionality of eddy fluxes of a property $A$ to its mean gradient:

$$
\left\langle A^{\prime} v_{j}^{\prime}\right\rangle=-K \frac{\partial\langle A\rangle}{\partial x_{j}}
$$

where $v_{j}$ is the velocity component, $x_{j}$ is a spatial coordinate, the $\langle$.$\rangle denotes some averaging, and primes$ mark eddy components (deviations from that average). The $K$ is the coefficient of transfer, in principle a tensor but for simplicity here assumed to be a scalar. The diffusive parameterization should only be applied for a conservative property.

There has been much interest in applying a diffusive parameterization to potential vorticity (PV) (Green 1970; Welander 1973; Marshall 1981). Importantly, if we use a diffusive parameterization of potential vorticity we do not need to separately parameterize eddy momentum and buoyancy fluxes, because they are already included in the eddy flux of potential vorticity. While the parameterization in terms of PV is well suited to approximations such as the quasigeostrophic formulation, primitive equation models widely used today are formulated in terms of the momentum equations and do not lend themselves as easily to a diffusive parameterization of PV.

Using a diffusive closure of eddy PV fluxes requires an integral constraint for the momentum budget known as the theorem of Bretherton to be introduced (Bretherton 1966; McWilliams et al. 1978; Marshall 1981) (see section 3). Some studies (Marshall 1981; Ivchenko 1984; Ivchenko et al. 1997, 2013, 2014a,b; Olbers et al. 2000) satisfy the momentum constraint by a suitable choice of diffusivity coefficient and others by inclusion of a so-called gauge term (Eden 2010).

McWilliams et al. (1978) and McWilliams and Chow (1981) demonstrated sharpening of zonal flow by PV mixing in an eddy-resolving quasigeostrophic zonal channel model. It was further demonstrated that using a diffusive parameterization of quasigeostrophic PV (QGPV) in a zonal channel can result in sharper and stronger currents (Ivchenko 1984; Ivchenko et al. 1997, 2014b), provided a spatially variable positive diffusion coefficient is specified, with local minima in regions occupied by jets. Dritschel and McIntyre
(2008) and Wood and McIntyre (2010) also performed theoretical studies of sharpening of zonal flows by PV diffusion.

Application of a diffusive parameterization of $\mathrm{PV}$ in a zonal reentrant channel [with application to the Antarctic Circumpolar Current (ACC)] has been studied in many papers both for domains with a flat bottom and domains with bottom topography included, but only for the zonally averaged case (Marshall 1981; Ivchenko 1984; Ivchenko et al. 1997, 2013, 2014a,b). Introduction of bottom topography creates a number of difficult complications (see Constantinou and Young 2017).

There are two major questions associated with application of a diffusive parameterization of $\mathrm{PV}$ in the presence of bottom topography:

1) Is the eddy PV diffusivity coefficient $K$ guaranteed to be positive? The coefficient $K$ varies in space and time. Its local value in some locations probably could occasionally be negative. However, can we be sure that the mean (averaged) value of $K$ is positive? Rhines and Young (1982) suggested that the eddy flux of PV is downgradient (i.e., positive eddy $\mathrm{PV}$ diffusivity) in an integral sense. There are not many analytical works that constrain the sign of PV diffusion. Abernathey et al. (2013) made an analysis based on a primitive equation model for a circumpolar channel. However, following Treguier et al. (1997) they calculated certain quasigeostrophic quantities, such as QGPV flux, background QGPV gradient, and corresponding diffusivity, using zonal averaging. The QGPV diffusivity is positive nearly everywhere, except near the surface, where the QG approximation is invalid. Birner et al. (2013), on the other hand, reveal a localized region of significant upgradient eddy PV fluxes on the poleward side of the subtropical free atmospheric jet core during the winter and spring seasons of both hemispheres. However, Birner et al. (2013) have noted that the net PV fluxes are downgradient when averaged over both the equatorward and poleward flanks of the jet.

In this study an analytical solution is provided that supports PV diffusivity being positive (in a domainaveraged sense). The assumption of a spatially constant eddy PV diffusivity is clearly unrealistic; however, it leads to a mathematically tractable problem and the solution provides insights that will remain applicable in the more general case.

2) How does one deal with the rotational (nondivergent) part of eddy PV flux? Eddy fluxes of PV comprise a rotational component and a divergent component: any 
vector $\mathbf{E}$ can be separated into divergent $\mathbf{E}_{\text {div }}$ and rotational $\mathbf{E}_{\text {rot }}$ parts (see next section).

The rotational component of the eddy flux of potential vorticity is likely to be substantial for a zonal channel with bottom topography (Sinha 1993). However, the rotational part does not directly influence the flow, because the divergence of the eddy flux appears in the PV equation and so the contribution of the rotational component is zero. The rotational part can, however, influence the flow by influencing the coefficient $K$ via the equation of eddy potential enstrophy (see section 2).

How can we determine the sign of $K$ for eddy diffusion of PV? One suggestion would be to use the results of eddy-resolving experiments with oceanic GCMs. We can calculate $\left\langle Q^{\prime} v_{j}^{\prime}\right\rangle(Q$ is $\mathrm{PV})$ and $\partial\langle Q\rangle / \partial x_{j}$ directly from model simulations, and then determine

$$
K=-\frac{\left\langle Q^{\prime} v_{j}^{\prime}\right\rangle}{\frac{\partial\langle Q\rangle}{\partial x_{j}}} .
$$

However, as already noted, the rotational part of $\left\langle Q^{\prime} v_{j}^{\prime}\right\rangle$ must be excluded from this calculation. A lack of inherited boundary condition makes separation of eddy fluxes of PV into divergent and rotational components for a finite domain with nonperiodic boundary conditions nonunique, as shown by Fox-Kemper et al. (2003).

Separation of the eddy PV flux into divergent and rotational components requires a specific boundary condition. Maddison et al. (2015) defined the divergent component of the PV flux by introducing a streamfunction tendency ("force function"). This is equivalent to a zero tangential component boundary condition (zero normal flux) and hence is not completely general. Mak et al. (2016) introduced a new method for diagnosing eddy diffusivity in a gauge-invariant fashion, which is independent of rotational flux components. This was achieved by seeking to match diagnosed and parameterized eddy force functions through an optimization procedure. The method was applied to a multilayer QG ocean gyre experiment, and it was demonstrated that the mean PV diffusivity over the horizontal domain is positive; however, robust locally negative diffusivity takes place even in the absence of rotational fluxes.

An alternative possibility is to determine the sign of the coefficient theoretically. In this study, we derive an analytical solution and construct an expression for kinetic energy, integrated over the whole domain, and use physical constraints on kinetic energy to demonstrate that the sign of $K$, interpreted as a domain-averaged PV diffusivity, must be positive. This is the first time that an analytical solution using a diffusive parameterization of PV has been derived for a barotropic quasigeostrophic zonal channel flow above zonally varying bottom topography. It is not, however, our intention to compare the relative merits of alternative eddy parameterizations.

The remainder of this paper is organized as follows. In section 2 we present the basic equations for quasigeostrophic barotropic flow and equations for a zonal channel geometry with bottom topography. In section 3 we formulate the generalized theorem of Bretherton. In section 4 we demonstrate an analytical solution for zonal flow, construct an expression for kinetic energy, and present results of our calculations for different types of topography. Section 5 consists of discussion and conclusions.

\section{Equations for zonal channel geometry including eddy parameterization}

The equation for barotropic quasigeostrophic vorticity can be written as follows:

$$
\frac{\partial q}{\partial t}+J(\Psi, q)=T+F_{B}+F_{H},
$$

where $q$ and $\Psi$ are the QGPV and streamfunction, respectively. Velocity $\mathbf{v}=(u, v)$ is related to the streamfunction by $u=-(\partial / \partial y) \Psi$ and $v=(\partial / \partial x) \Psi$, where $u$ and $v$ are the velocity components in the zonal $(x)$ and meridional $(y)$ directions. The $J(A, B)$ is the Jacobian operator: $J(A, B)=-(\partial A / \partial y)(\partial B / \partial x)+(\partial A / \partial x)(\partial B / \partial y)$. The $T, F_{B}$, and $F_{H}$ are the external forcing (wind stress), bottom friction, and horizontal friction, respectively.

The QGPV $q$ in barotropic flow represents the sum of relative vorticity, planetary vorticity, and the topographic term, given by

$$
q=\nabla^{2} \Psi+f+\frac{f_{0}}{H} B
$$

where Coriolis parameter $f=f_{0}+\beta y$. The $f_{0}$ and $\beta$ denote its value at a reference latitude and its meridional gradient, respectively. The $B$ is the deviation of bottom topography from a constant depth $H$.

Bottom topography substantially complicates the dynamics. The streamfunction exhibits nonzonal meanders near topographic obstacles (McWilliams et al. 1978), and therefore it is necessary to perform spatial averaging not for the whole zonal length, but for only part of it. The averaged equations depend on both zonal and meridional directions, which creates much more mathematical complexity compared to the fully zonally averaged 
case, but they remain analytically tractable as we will demonstrate.

To understand eddy dynamics it is important to consider the equation for quasigeostrophic eddy potential enstrophy (QGEPE). To derive the QGEPE equation, we define a time average and partial zonal average of an arbitrary variable, denoted by an overbar, $\bar{A}$, and a deviation from this average, denoted by a superscript prime, $A^{\prime}=A-\bar{A}$ :

$$
\overline{A(x, t)}=\frac{1}{2 \delta_{x} T} \int_{t}^{t+T} \int_{x-\delta_{x}}^{x+\delta_{x}} A\left(x^{\prime}, t^{\prime}\right) d x^{\prime} d t^{\prime},
$$

where $\delta_{x}$ is the average scale for zonal coordinate and $T$ is the averaging time. Note that the partial zonal and time average is a more appropriate type of average for the zonal channel domain with variable topography than a time-only average, since bottom topography being time independent cannot contribute to the eddy topographic form stress in the case of a time average (see section 3).

We average Eq. (3), subtract the resulting equation from Eq. (3), multiply by $q^{\prime}$, and average once again to obtain the following:

$$
\begin{aligned}
\frac{1}{2} \frac{\overline{\partial q^{\prime 2}}}{\partial t}=0= & -\frac{1}{2} \operatorname{div}\left(\overline{\mathbf{v}} \overline{q^{\prime 2}}\right)-\overline{\mathbf{v}^{\prime} q^{\prime}} \cdot \nabla \bar{q}-\frac{1}{2} \operatorname{div}\left(\overline{\mathbf{v}^{\prime} q^{\prime 2}}\right) \\
& +\overline{T^{\prime} q^{\prime}}+\overline{F_{B}^{\prime} q^{\prime}}+\overline{F_{H}^{\prime} q^{\prime}} .
\end{aligned}
$$

Equation (6) is a well-known equation for quasigeostrophic eddy potential vorticity QGEPE (see, e.g., Vallis 2006). The terms on the RHS of Eq. (6) represent redistribution by the mean flow, generation, redistribution by eddies, input from external sources, and dissipation by bottom and horizontal friction of QGEPE, respectively.

If we now specify the domain as a zonal reentrant channel and integrate Eq. (6) over the whole domain $S$, then the terms responsible for redistribution (i.e., the first and the third terms on the RHS) drop out because of boundary conditions on the solid walls and periodicity. A similar equation was derived by Constantinou and Young (2017). We assume that the external forcing is stationary $\left(T^{\prime}=0\right)$, and therefore the fourth term on the RHS is zero, which leads to

$$
\begin{aligned}
\int_{(S)} \frac{1}{2} \frac{\overline{\partial q^{\prime 2}}}{\partial t} d S=0= & -\int_{(S)} \overline{\mathbf{v}^{\prime} q^{\prime}} \cdot \nabla \bar{q} d S \\
& +\int_{(S)}\left(\overline{F_{B}^{\prime} q^{\prime}}+\overline{F_{H}^{\prime} q^{\prime}}\right) d S .
\end{aligned}
$$

Equation (7) represents the balance between the generation of the QGEPE (the first term on the
RHS) and dissipation by bottom and horizontal friction. The dissipation terms measure the integral loss of enstrophy, and therefore the integral of the generation should be positive. Numerical experiments with eddy-resolving models demonstrate that the generation term locally takes both signs (Sinha 1993; J.-O. Wolff 2017, personal communication). However, the integral over the domain must be positive.

The eddy flux $\overline{\mathbf{v}^{\prime} q^{\prime}}$ comprises two parts, the divergent flux $\mathbf{E}_{\text {div }}$ and rotational flux $\mathbf{E}_{\text {rot }}$ :

$$
\overline{\mathbf{v}^{\prime} q^{\prime}}=\mathbf{E}_{\mathrm{div}}+\mathbf{E}_{\text {rot }},
$$

where $\operatorname{curl}_{z}\left(\mathbf{E}_{\text {div }}\right)=0, \operatorname{div}\left(\mathbf{E}_{\text {rot }}\right)=0$, and $\operatorname{curl}_{z} \mathbf{E}_{\text {div }}=$ $\left.\partial \mathbf{E}_{\mathrm{div}}\right|_{y} / \partial x-\left.\partial \mathbf{E}_{\mathrm{div}}\right|_{x} / \partial y$.

The traditional diffusive parameterization of QGPV can be written as

$$
\overline{\mathbf{v}^{\prime} q^{\prime}}=-k \nabla \bar{q},
$$

and the term representing generation of QGEPE in Eqs. (6) and (7) is

$$
\overline{\mathbf{v}^{\prime} q^{\prime}} \cdot \nabla \bar{q}=-k|\nabla \bar{q}|^{2},
$$

where $k$ is the coefficient of eddy diffusivity of QGPV.

The equation for mean QGPV [Eq. (3)] for the stationary (time-independent case) takes the following form:

$\bar{u} \frac{\partial \bar{q}}{\partial x}+\bar{v} \frac{\partial \bar{q}}{\partial y}+\frac{\partial \overline{u^{\prime} q^{\prime}}}{\partial x}+\frac{\partial \overline{v^{\prime} q^{\prime}}}{\partial y}=\frac{1}{H} \operatorname{curl}_{z} \bar{\tau}-\epsilon \operatorname{curl}_{z} \overline{\mathbf{v}}$.

We specify QGPV input due to surface wind stress in the traditional manner: $T=(1 / H) \operatorname{curl}_{z} \bar{\tau}$, where $\boldsymbol{\tau}$ represents tangential wind stress divided by the water density, and $F_{B}=-\epsilon \operatorname{curl}_{z} \overline{\mathbf{v}}$, bottom friction, where $\epsilon$ is a coefficient of bottom friction. Horizontal friction is disregarded.

Using Eq. (9), Eq. (11) becomes

$$
\begin{gathered}
\bar{u} \frac{\partial \bar{q}}{\partial x}+\bar{v} \frac{\partial \bar{q}}{\partial y}-\frac{\partial}{\partial x} k \frac{\partial \bar{q}}{\partial x}-\frac{\partial}{\partial y} k \frac{\partial \bar{q}}{\partial y} \\
=\frac{1}{H} \operatorname{curl}_{z} \bar{\tau}-\epsilon \operatorname{curl}_{z} \overline{\mathbf{v}} .
\end{gathered}
$$

We proceed to solve Eq. (12) by expanding each of the variables into a Fourier series. We assume that the bottom topography has meridional length scale comparable with the width of the channel $L$ and retain only the first term in the Fourier decomposition of topography in the meridional direction. In the zonal direction we allow a variety of length scales for bottom topography, both large 
and small scales, and impose no limit to the number of terms in the Fourier series. Such detailed representation in the zonal direction is important for zonal flows, because it allows better representation of the nonviscous bottom form stress, which is important for balancing the external forcing (wind stress) and results in a substantial decrease of the zonal transport, compared with the flat-bottom case (Munk and Palmén 1951; McWilliams et al. 1978; Wolff et al. 1991).

We assume no mass flux through the solid walls:

$$
\left.\bar{v}\right|_{y=0, L}=0 \text {. }
$$

We also assume no QGPV flux through the walls:

$$
\left.\overline{v^{\prime} q^{\prime}}\right|_{y=0, L}=-k \frac{\partial \bar{q}}{\partial y}=0 .
$$

The condition defined by Eq. (14) can only be satisfied if $k$ is zero on the solid walls, because of the presence of the planetary vorticity gradient $\beta$ in the expression for the meridional gradient of QGPV; that is, on the boundaries $\partial \bar{q} / \partial y$ cannot be zero, and therefore $k$ must be zero. Hence, we specify the following form of the coefficient $k$ :

$$
k=k_{0}\left[1+e^{(-L / \Delta)}-e^{(-y / \Delta)}-e^{(y-L) / \Delta)}\right],
$$

where $\Delta \ll L$ and $k_{0}$ is a constant. The $k$ is almost constant in the domain but quickly drops to zero on the sidewalls (see Fig. 1). The difference between $k$ and $k_{0}$ at any point of the domain will be small by choosing $\Delta$ to be small enough, except on the solid boundaries, where $k=0$.

\section{Generalized theorem of Bretherton}

In a zonal channel with a flat bottom the total (domain averaged) meridional eddy fluxes of QGPV must be zero, to satisfy the mean zonal momentum budget (Bretherton 1966). This statement, known as the theorem of Bretherton (McWilliams et al.1978), provides an integral constraint for coefficients of QGPV diffusivity (Marshall 1981; Ivchenko 1984). In a zonal channel with variable bottom topography this statement can be generalized using zonal and time averaging (Ivchenko 1987; Ivchenko et al. 2013, 2014a), which allows us to include an important topographic form stress in the mean zonal momentum balance. In this study we find a solution depending on both meridional and zonal coordinates and therefore use partial zonal and time averages [Eq. (5)]. Let us first calculate the eddy QGPV flux by multiplying

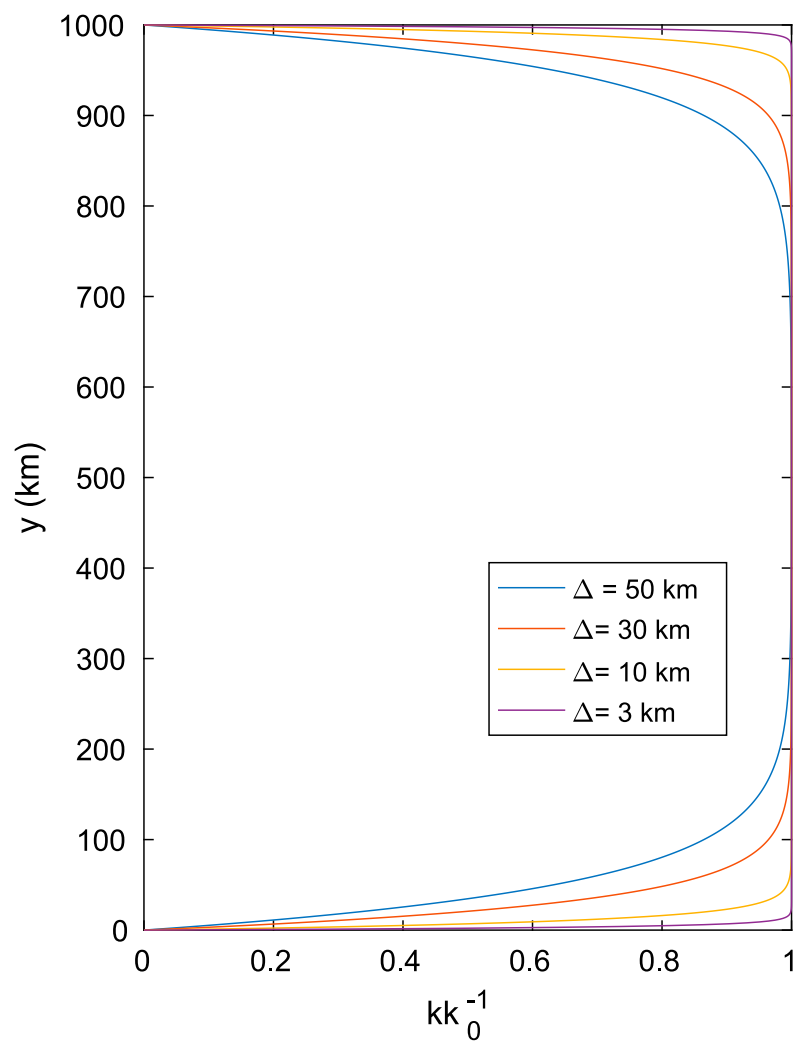

FIG. 1. Meridional profile of the QGPV diffusion coefficient $k$ normalized by $k_{0}$.

$q$ [see Eq. (4)] by $v^{\prime}$, taking an average and integrating over the whole basin:

$$
\begin{aligned}
\int_{0}^{L} \int_{0}^{L_{x}} \overline{v^{\prime} q} d x d y= & \int_{0}^{L} \int_{0}^{L_{x}}\left[\overline{\boldsymbol{v}^{\prime}\left(\frac{\partial v}{\partial x}-\frac{\partial u}{\partial y}\right)}\right. \\
& \left.+f \overline{\boldsymbol{v}^{\prime}}+\frac{f_{0}}{\boldsymbol{v}^{\prime} B}\right] d x d y
\end{aligned}
$$

where $L_{x}$ is the zonal length of the channel. The first term in the RHS of Eq. (16) can be transformed, using the continuity equation:

$$
\begin{aligned}
& \int_{0}^{L} \int_{0}^{L_{x}} \overline{v^{\prime}\left(\frac{\partial v}{\partial x}-\frac{\partial u}{\partial y}\right)} d x d y \\
& \quad=\int_{0}^{L} \int_{0}^{L_{x}}\left(\frac{1}{2} \frac{\partial \overline{v^{\prime 2}}}{\partial x}-\frac{\partial \overline{u^{\prime} v^{\prime}}}{\partial y}-\frac{1}{2} \frac{\partial \overline{u^{\prime 2}}}{\partial x}\right) d x d y .
\end{aligned}
$$

The first and the third terms in the RHS of Eq. (17) drop to zero because of periodicity, and the second term drops to zero because there is no flux through the solid walls. It is obvious that the second term in the RHS of Eq. (16) is zero, so using $\overline{v^{\prime} q}=\overline{v^{\prime} q^{\prime}}$ [Eq. (16)] can be written as follows: 


$$
\int_{0}^{L} \int_{0}^{L_{x}} \overline{\boldsymbol{v}^{\prime} q^{\prime}} d x d y=\int_{0}^{L} \int_{0}^{L_{x}} \overline{f_{0}} \overline{\boldsymbol{v}^{\prime} B^{\prime}} d x d y .
$$

This means that redistribution of QGPV by eddies (LHS) is balanced by topographic form stress (RHS), exerted by eddies.

If we introduce a diffusive parameterization [Eq. (9)] together with the expression for $k$ [Eq. (15)] into the LHS of Eq. (18) we obtain

$$
\begin{aligned}
\int_{0}^{L} \int_{0}^{L_{x}} \overline{v^{\prime} q^{\prime}} d x d y= & -\beta k_{0} L_{x} L\left\{1+e^{(-L / \Delta)}\right. \\
& \left.-\frac{2 \Delta\left[1-e^{(-L / \Delta)}\right]}{L}\right\}
\end{aligned}
$$

The expression in curly brackets is close to unity. Therefore, Eq. (18) becomes

$$
-\beta k_{0}=\frac{f_{0}}{H} \frac{1}{L_{x} L} \int_{0}^{L} \int_{0}^{L_{x}} \overline{v^{\prime} B^{\prime}} d x d y .
$$

The $\beta k_{0}$ term appeared in studies by Welander (1973), Killworth (1997), Eden (2010), and many others. Equation (20) provides a clear physical explanation of this term: eddy topographic form stress is exerted by parameterized eddies. Note that using only time averaging without partial zonal averaging would lead to the eddy flux associated with the topographic part of the QGPV dropping to zero [bottom topography is time independent; hence the RHS of Eq. (20) is zero], and this would imply that the coefficient $k_{0}$ has to be zero.

\section{Analytical solution for zonal flow}

\section{a. Model setup}

We now assume that the solution for Eq. (12) consists of a constant zonal flow with (unknown) velocity $U$ and streamfunction $\Phi$ multiplied by the first meridional Fourier mode:

$$
\bar{\Psi}=-U y+\Phi(x) \sin (\pi y / L) .
$$

We follow Charney et al. (1981), who used a similar technique for an atmospheric flow in a zonal channel. We represent the topographic term $B$ in the following form:

$$
B=h(x) \sin (\pi y / L) .
$$

The net zonal transport across the channel depends only on $U$, because $\Phi(x) \sin [(\pi y) / L]$ does not affect the net transport, although it does affect the zonal velocity locally because of topography and diffusion of the QGPV. We specify the surface wind stress $\tau=\left(\tau_{x}, \tau_{y}\right)$, with $\tau_{y}=0$, and let the zonal component of wind stress be proportional to the sine of latitude with the maximum value in the center of the channel and zero on the walls; that is, $\tau_{x}=\tau_{0} \sin [(\pi y) / L]$.

Using Eqs. (21)-(22) the velocity and the gradient of potential vorticity can be easily calculated. So, Eq. (12) after transformation can be rewritten in this form:

$$
\begin{aligned}
& \sin (\pi y / L)\left(U \Phi_{x x x}-U \frac{\pi^{2}}{L^{2}} \Phi_{x}+\beta \Phi_{x}+\frac{f_{0}}{H} U h_{x}-k \Phi_{x x x x}\right. \\
& \left.+k \frac{\pi^{2}}{L^{2}} \Phi_{x x}-k \frac{f_{0}}{H} h_{x x}\right)+\sin (\pi y / L) \cos (\pi y / L) \\
& \quad \times\left[-\frac{\pi}{L} \Phi \Phi_{x x x}-\left(\frac{\pi}{L}\right) \frac{f_{0}}{H}\left(\Phi h_{x}-\Phi_{x} h\right)+\frac{\pi}{L} \Phi_{x} \Phi_{x x}\right] .
\end{aligned}
$$

Subscripts $x\left(\Phi_{x}, h_{x}\right.$ and so on) mark zonal derivatives, and the number of subscripts correspond to the derivative order: $\Phi_{x}=\partial \Phi / \partial x, \Phi_{x x}=\partial^{2} \Phi / \partial x^{2}$, and so on. In Eq. (23) the term of meridional gradient of the meridional flux of eddy QGPV is retained without transformation since it simplifies after a meridional integration, which we carry out in section $4 b$.

\section{b. Momentum balance}

To simplify the QGPV equation and remove the $y$ dependence we integrate Eq. (23) meridionally between 0 and $L$, resulting in

$$
\begin{aligned}
& U\left(\Phi_{x x x}-\frac{\pi^{2}}{L^{2}} \Phi_{x}\right)+U \frac{f_{0}}{H} h_{x}+\Phi_{x} \beta \\
& -k_{0}\left(\Phi_{x x x x}-\frac{\pi^{2}}{L^{2}} \Phi_{x x}+\frac{f_{0}}{H} h_{x x}\right)\left\{1-\frac{\pi^{2} \Delta^{2}\left[1+e^{(-L / \Delta)}\right]}{\left(L^{2}+\pi^{2} \Delta^{2}\right)}\right\} \\
& +\epsilon \Phi_{x x}-\epsilon\left(\frac{\pi}{L}\right)^{2} \Phi=0 .
\end{aligned}
$$

To derive this equation we assume that the eddy flux through the solid walls is zero $\overline{v^{\prime} q^{\prime}}=0$ [boundary condition in Eq. (14)]. We also make use of the property that terms that are proportional to $\sin [(\pi y) / L] \cos [(\pi y) / L]$ integrate to zero.

We can obtain a further useful relationship by returning to Eq. (23), multiplying by $\cos [(\pi y) / L]$ and integrating meridionally between the solid boundaries. There is an important term $\int_{0}^{L} \cos (\pi y / L)(\partial / \partial y)\left(\overline{v^{\prime} q^{\prime}}\right) d y$, which after substitution of Eqs. (9) and (15), becomes 


$$
\begin{aligned}
& \int_{0}^{L} \cos [(\pi y) / L] \frac{\partial}{\partial y} k \frac{\partial \bar{q}}{\partial y} d y \\
& \quad=2 \beta k_{0}\left\{1+e^{(-L / \Delta)}-\frac{\pi^{2} \Delta^{2}\left[1+e^{(-L / \Delta)}\right]}{\left(L^{2}+\pi^{2} \Delta^{2}\right)}\right\} .
\end{aligned}
$$

So Eq. (23) multiplied by $\cos [(\pi y) / L]$ and integrated meridionally yields

$$
\begin{aligned}
& \Phi \Phi_{x x x}-\Phi_{x} \Phi_{x x}+\frac{f_{0}}{H}\left(\Phi h_{x}-\Phi_{x} h\right) \\
& \quad+3 \beta k_{0}\left\{1+e^{(-L / \Delta)}-\frac{\pi^{2} \Delta^{2}\left[1+e^{(-L / \Delta)}\right]}{\left(L^{2}+\pi^{2} \Delta^{2}\right)}\right\}=\frac{3 \tau_{0} \pi}{4 H} .
\end{aligned}
$$

Note that because $\Delta \ll L$ the expressions in the curly brackets in Eqs. (24)-(26) are very close to unity, so we approximate them as unity with negligible error.

We integrate Eq. (26) with respect to $x$ between 0 and $L_{x}$ to eliminate the zonal dependence and elucidate the zonal momentum balance:

$$
\begin{aligned}
& \int_{0}^{L_{x}}\left[\Phi \Phi_{x x x}-\Phi_{x} \Phi_{x x}+\frac{f_{0}}{H}\left(\Phi h_{x}-\Phi_{x} h\right)\right] d x+3 \beta k_{0} L_{x} \\
& \quad=\frac{3 L_{x} \tau_{0} \pi}{4 H}
\end{aligned}
$$

It is easy to show that

$$
\int_{0}^{L_{x}}\left(\Phi \Phi_{x x x}-\Phi_{x} \Phi_{x x}\right) d x=0,
$$

because of the periodicity of the channel. Also,

$$
\int_{0}^{L_{x}}\left(\Phi h_{x}\right) d x=-\int_{0}^{L_{x}}\left(\Phi_{x} h\right) d x .
$$

Thus, Eq. (27) can be rewritten as follows:

$$
\frac{3 L_{x} \tau_{0} \pi}{8}=f_{0} \int_{0}^{L_{x}}\left(\Phi h_{x}\right) d x+\frac{3 \beta k_{0} L_{x} H}{2} .
$$

Equation (30) describes the stationary momentum balance. On the LHS there is a contribution from wind stress. The first term on the RHS, that is, $f_{0} \int_{0}^{L_{x}} \Phi h_{x} d x$, is the topographic form stress exerted by the mean flow, since it is an integral of the product of a pressure (equal to streamfunction times Coriolis parameter $f_{0}$ ) and the zonal gradient of bottom topography. The second term on the RHS is a topographic form stress exerted by unresolved parameterized eddies on the bottom topography [see Eq. (20)].

\section{c. Energy balance}

We form the energy power integral by multiplying the $y$-integrated QGPV [Eq. (24)] with $\Phi(x)$ and integrate over $x$. After some manipulation we obtain

$$
\begin{aligned}
& U \frac{f_{0}}{H} \int_{0}^{L_{x}}\left(\Phi h_{x}\right) d x-k_{0} \int_{0}^{L_{x}}\left(\Phi_{x x}\right)^{2} d x \\
& -k_{0} \frac{\pi^{2}}{L^{2}} \int_{0}^{L_{x}}\left(\Phi_{x}\right)^{2} d x+k_{0} \frac{f_{0}}{H} \int_{0}^{L_{x}} \Phi_{x} h_{x} d x \\
& -\epsilon \int_{0}^{L_{x}}\left(\Phi_{x}\right)^{2} d x-\epsilon \frac{\pi^{2}}{L^{2}} \int_{0}^{L_{x}}(\Phi)^{2} d x=0 .
\end{aligned}
$$

Substitution of Eq. (30) into Eq. (31) after transformation yields an equation of balance of kinetic energy of the "perturbed" flow $E=E_{U}+E_{V}$, where

$$
\begin{aligned}
E_{U} & =\frac{1}{L_{x} L} \int_{0}^{L_{x}} \int_{0}^{L} \frac{(\bar{u}-U)^{2}}{2} d x d y, \text { and } \\
E_{V} & =\frac{1}{L_{x} L} \int_{0}^{L_{x}} \int_{0}^{L} \frac{\bar{v}^{2}}{2} d x d y .
\end{aligned}
$$

For a steady state, the kinetic energy balance equation of the perturbed flow can be written as

$$
\{E, \tau\}=\{E, k\}+\{E, h\}+\{E, \epsilon\}+\{E, \beta\} .
$$

The terms in Eq. (34) are as follows:

$$
\{E, \tau\}=\frac{3 \pi}{8 H} U \tau_{0}
$$

represents generation of kinetic energy by wind stress;

$$
\{E, k\}=\frac{k_{0}}{L_{x}} \int_{0}^{L_{x}}\left[\left(\Phi_{x x}\right)^{2}+\frac{\pi^{2}}{L^{2}}\left(\Phi_{x}\right)^{2}\right] d x>0, \quad\left(\text { if } k_{0}>0\right)
$$

represents dissipation of energy by mixing of QGPV; and

$$
\{E, h\}=-\frac{k_{0}}{L_{x}} \int_{0}^{L_{x}}\left(\frac{f_{0}}{H} \Phi_{x} h_{x}\right) d x>0
$$

represents dissipation by QGPV mixing linked with bottom topography and is positive because of conservation of QGPV. In the mainly eastward flow there is an equatorward (i.e., $\Phi_{x}>0$ ) deflection if the motion is uphill $\left(h_{x}>0\right)$ and a poleward (i.e., $\Phi_{x}<0$ ) deflection if the motion is downhill $\left(h_{x}<0\right)$. So, $-\left(f_{0} / H\right) \int_{0}^{L_{x}} \Phi_{x} h_{x} d x>0$, since the Coriolis parameter is negative in the Southern Hemisphere. Note that the sign of this term is positive in the Northern Hemisphere as 
well, since not only is the Coriolis parameter of opposite sign, but the "equatorward/poleward" motions are also reversed. The term

$$
\{E, \epsilon\}=+\frac{\epsilon}{L_{x}} \int_{0}^{L_{x}}\left[\left(\Phi_{x}\right)^{2}+\frac{\pi^{2}}{L^{2}}(\Phi)^{2}\right] d x>0
$$

represents dissipation by bottom friction. The last term,

$$
\{E, \beta\}=\frac{3 U k_{0} \beta}{2}>0 \quad\left(\text { if } k_{0}>0\right),
$$

is proportional to $U, k_{0}$, and $\beta$, and using Eq. (20) could be rewritten as

$$
\{E, \beta\}=-\frac{3}{2} U \frac{f_{0}}{H} \frac{1}{L_{x} L} \int_{0}^{L} \int_{0}^{L_{x}} \overline{v^{\prime} B^{\prime}} d x d y
$$

and is a sink of kinetic energy due to topographic form stress exerted by parameterized eddies. A conceptually similar term was introduced by Carnevale and Frederiksen (1987) in their study of periodic flow on a $\beta$ plane. They consider how best to model the interaction of "small" scale (synoptic) flow features with large- or basin-scale features. Carnevale and Frederiksen (1987) emphasize the role of the integral invariants. They demonstrated that the rate of change of energy in the "small scales" is given by a term similar to the RHS of Eq. (40) [see the RHS of Eq. (5.5) of the paper by Carnevale and Frederiksen (1987)].

The three terms $\{E, k\},\{E, h\}$, and $\{E, \beta\}$ are proportional to $k_{0}$ and represent dissipation of energy only if $k_{0}>0$. If $k_{0}<0$ all these terms are physically incorrect.

\section{d. Analytical solution}

To obtain an analytical solution let us write $\Phi$ and topography $h(x)$ as Fourier series:

$$
\begin{array}{cc}
\Phi & =\sum_{n} a_{n} \cos \left(\frac{2 n \pi x}{L_{x}}\right)+\sum_{n} b_{n} \sin \left(\frac{2 n \pi x}{L_{x}}\right), \quad \text { and } \\
h & =\sum_{n} c_{n} \cos \left(\frac{2 n \pi x}{L_{x}}\right)+\sum_{n} d_{n} \sin \left(\frac{2 n \pi x}{L_{x}}\right),
\end{array}
$$

where $a_{n}$ and $b_{n}$ are unknown constants, and $c_{n}$ and $d_{n}$ are constants relating to the prescribed topography. The $n$ is the index of each mode used in the Fourier expansion.

Substituting Eqs. (41) and (42) in the meridionally integrated QGPV [Eq. (24)] and equating coefficients of $\sin \left[(2 n \pi x) / L_{x}\right]$ and $\cos \left[(2 n \pi x) / L_{x}\right]$ results in two equations:

$$
\begin{aligned}
& a_{n}\left[U M^{(n)}-\beta \frac{2 \pi}{L_{x}} n\right]-b_{n} N^{(n)}-c_{n} U \frac{f_{0}}{H} \frac{2 \pi}{L_{x}} n \\
& \quad+d_{n} k_{0} \frac{f_{0}}{H}\left(\frac{2 \pi}{L_{x}}\right)^{2} n^{2}=0, \text { and } \\
& -a_{n} N^{(n)}+b_{n}\left[-U M^{(n)}+\beta \frac{2 \pi}{L_{x}} n\right]+c_{n} k_{0} \frac{f_{0}}{H}\left(\frac{2 \pi}{L_{x}}\right)^{2} n^{2} \\
& \quad+d_{n} U \frac{f_{0}}{H} \frac{2 \pi}{L_{x}} n=0
\end{aligned}
$$

where

$$
\begin{aligned}
& M^{(n)}=\left(\frac{2 \pi}{L_{x}}\right)^{3} n^{3}+\frac{2 \pi^{3}}{L^{2} L_{x}} n, \quad \text { and } \\
& N^{(n)}=k_{0}\left(\frac{2 \pi}{L_{x}}\right)^{4} n^{4}+k_{0} \frac{4 \pi^{4}}{L^{2} L_{x}^{2}} n^{2}+\epsilon\left(\frac{2 \pi}{L_{x}}\right)^{2} n^{2}+\epsilon\left(\frac{\pi}{L}\right)^{2} .
\end{aligned}
$$

Solution of the two algebraic equations [Eqs. (43) and (44)] yields the following:

$$
\begin{aligned}
a_{n}= & \frac{S_{0}^{(n)}+U S_{1}^{(n)}+U^{2} S_{2}^{(n)}}{R_{0}^{(n)}+U R_{1}^{(n)}+U^{2} R_{2}^{(n)}}, \text { and } \\
b_{n}= & \frac{1}{N^{(n)}}\left\{\frac{U M^{(n)}\left[S_{0}^{(n)}+U S_{1}^{(n)}+U^{2} S_{2}^{(n)}\right]}{R_{0}^{(n)}+U R_{1}^{(n)}+U^{2} R_{2}^{(n)}}\right. \\
& -\frac{\beta \frac{2 \pi}{L_{x}} n\left[S_{0}^{(n)}+U S_{1}^{(n)}+U^{2} S_{2}^{(n)}\right]}{R_{0}^{(n)}+U R_{1}^{(n)}+U^{2} R_{2}^{(n)}} \\
& \left.-U c_{n} \frac{f_{0}}{H} \frac{2 \pi}{L_{x}} n+d_{n} k_{0} \frac{f_{0}}{H}\left(\frac{2 \pi}{L_{x}}\right)^{2} n^{2}\right\}
\end{aligned}
$$

New parameters $R_{0}^{(n)}, R_{1}^{(n)}, R_{2}^{(n)}, S_{0}^{(n)}, S_{1}^{(n)}$, and $S_{2}^{(n)}$ have been introduced. Their values can be seen in the appendix.

Coefficients $a_{n}$ and $b_{n}$ in Eqs. (47) and (48) still contain the unknown mean zonal velocity $U$. To find $U$ we substitute the Fourier series of Eqs. (41) and (42) in the zonal momentum balance equation [Eq. (30)]. After some manipulation we obtain

$$
\tau_{0}=\frac{8 f_{0}}{3 L_{x}} \sum_{n} n\left(a_{n} d_{n}-b_{n} c_{n}\right)+\frac{4 \beta k_{0} H}{\pi} .
$$

The method of solution to obtain $U$ is presented in the appendix.

The zonal flow is perturbed by the presence of topography and diffusion of QGPV. In the case of a flat bottom (i.e., $c_{n}=d_{n}=0$ ) the motion is unperturbed, since $S_{0}^{(n)}=S_{1}^{(n)}=S_{2}^{(n)}=0$ [see Eqs. (A4)-(A6)]. 
This analytical solution is possible because only a single meridional component of the bottom topography $B$ is retained. In the case of a more general expression of $B$ it would be much more difficult to obtain an analytical solution because of greatly increased mathematical complexity.

The expressions for the unknowns $a_{n}, b_{n}$, and $U$ in Eqs. (47)-(49) constitute an analytical solution for Eq. (23). There is no truncation error, since only the Fourier modes represented in the bottom topography contribute. Note that as long as the modulus of the amplitudes $c_{n}$ and $d_{n}$ of the Fourier topographic modes are finite, then

$$
\lim _{n \rightarrow \infty} a_{n}=\lim _{n \rightarrow \infty} b_{n}=0 .
$$

We evaluate the solution for a number of cases with different topography and different $k_{0}$ using parameter values relevant to the Southern Ocean: channel length $L_{x}=4 \times 10^{6} \mathrm{~m}$ and width $10^{6} \mathrm{~m}$; reference depth $5 \times 10^{3} \mathrm{~m}$; Coriolis parameter $f_{0}=-10^{-4} \mathrm{~s}^{-1}$ and $\beta=1.4 \times 10^{-11} \mathrm{~m}^{-1} \mathrm{~s}^{-1}$; and $\tau_{0}=10^{-4} \mathrm{~m}^{2} \mathrm{~s}^{-2}$. We illustrate the streamfunction for three cases: case 1 specifies the topography as $c_{3}=300 \mathrm{~m}$; case 2 specifies $c_{1}=300 \mathrm{~m}$ and $d_{1}=300 \mathrm{~m}$; case 3 specifies $c_{2}=300 \mathrm{~m}$ and $d_{5}=300 \mathrm{~m}$ (see Figs. 2-4). Here and later the topographic Fourier coefficients whose values are not explicitly stated are set to zero.

All cases demonstrate eastward mean flow, with streamlines deflected by topographic features (see Figs. 2-4). In these cases the bottom topography and coefficient $k_{0}$ vary. Because they obstruct the flow, the topographic features (both the amplitude and length in the zonal direction) substantially affect the net zonal volume transport (Figs. 5-7, upper panels). The streamfunctions for given topography look similar for different coefficients, but not the total zonal transport, which decreases linearly from the case with $k_{0}=0$ to the highest allowed coefficient. As we demonstrate above, the coefficient $k_{0}$ must be positive and according to the kinetic energy balance in Eq. (34) should be less than $k_{\max }=\pi \tau_{0} /(4 \beta H)$, since

$$
\{E, \tau\}-\{E, \beta\}>0 .
$$

Under our selected parameters, $\pi \tau_{0} /(4 \beta H)=1.12 \times$ $10^{3} \mathrm{~m}^{2} \mathrm{~s}^{-1}$. However, our solutions demonstrate that the actual maximum value $k_{\max }^{\text {eff }}$ is less than this; that is,

$$
k_{\max }^{\text {eff }}<k_{\max }=\pi \tau_{0} /(4 \beta H)
$$

(see the middle panels in Figs. 5-7). This is because $k_{\max }^{\text {eff }}$ depends not only on Eq. (51) but on the other terms on the RHS of Eq. (34) as well.
Under prescribed topography the maximum transport corresponds to $k_{0}=0$, which varies substantially (depending on topography). The highest transports are $300.5,445.0$, and $115.0 \mathrm{~Sv}\left(1 \mathrm{~Sv} \equiv 10^{6} \mathrm{~m}^{3} \mathrm{~s}^{-1}\right)$ in cases $1-3$, respectively.

Being under the same external forcing (wind stress) the difference in transport occurs because of different bottom topography in these cases. The main momentum sink is the topographic form stress. This term strongly depends on the amplitudes and wavenumbers of the nonzero Fourier modes making up the topography. To quantify this dependence we introduce a new integral measure $D$ of the roughness of the topography [rms of $(\partial B / \partial x)]$ :

$$
D=\sqrt{\frac{1}{L L_{x}} \int_{0}^{L} \int_{0}^{L_{x}}\left(\frac{\partial B}{\partial x}\right)^{2} d x d y} .
$$

Substituting Eqs. (22) and (42) into Eq. (53) with an appropriate Fourier transformation we obtain

$$
D=\frac{\pi}{L_{x}} \sqrt{\sum_{n}\left[n^{2}\left(c_{n}^{2}+d_{n}^{2}\right)\right]} .
$$

This integral scale of roughness is dimensionless and depends on the mode index $n$ and the amplitude of the topography $c_{n}, d_{n}$. In Fig. 8 we plot points representing our calculations of transport for the same wind stress $\left(\tau_{0}=10^{-4} \mathrm{~m}^{2} \mathrm{~s}^{-2}\right)$ and $k_{0}=0$, but for the various realizations of bottom topography and a fitting curve that is seen to resemble a hyperbola. If $D<3 \times 10^{-4}$ there is a large variation of transport for a small variation of $D$. Small values of $D$ correspond to low amplitudes of topography together with small mode index $n$ (i.e., smooth topography). When $D>3 \times 10^{-4}$ there is an approximately linear relation between transport and $D$.

All the terms on the RHS of Eq. (34) are positive (Figs. 5-7, middle panels) and contribute to balancing the source of kinetic energy $(\{E, \tau\})$. When $k_{0}=0$ there is a balance between generation of kinetic energy by wind stress and dissipation by bottom friction; that is, $\{E, \tau\}=\{E, \epsilon\}$.

For small values of $k_{0}$, the bottom friction dominates the other terms. However, with increasing $k_{0}$ the terms $\{E, h\}$ and $\{E, \beta\}$ increase, representing dissipation linked with topography and the sink due to topographic form stress, respectively. These provide a substantial contribution to balancing the wind stress term. In all cases, the term $\{E, k\}$ representing dissipation by QGPV mixing remains small. The $\{E, k\}$ is not directly linked with topography in contrast to $\{E, h\}$. The highest values of kinetic energy $E$ and its components 

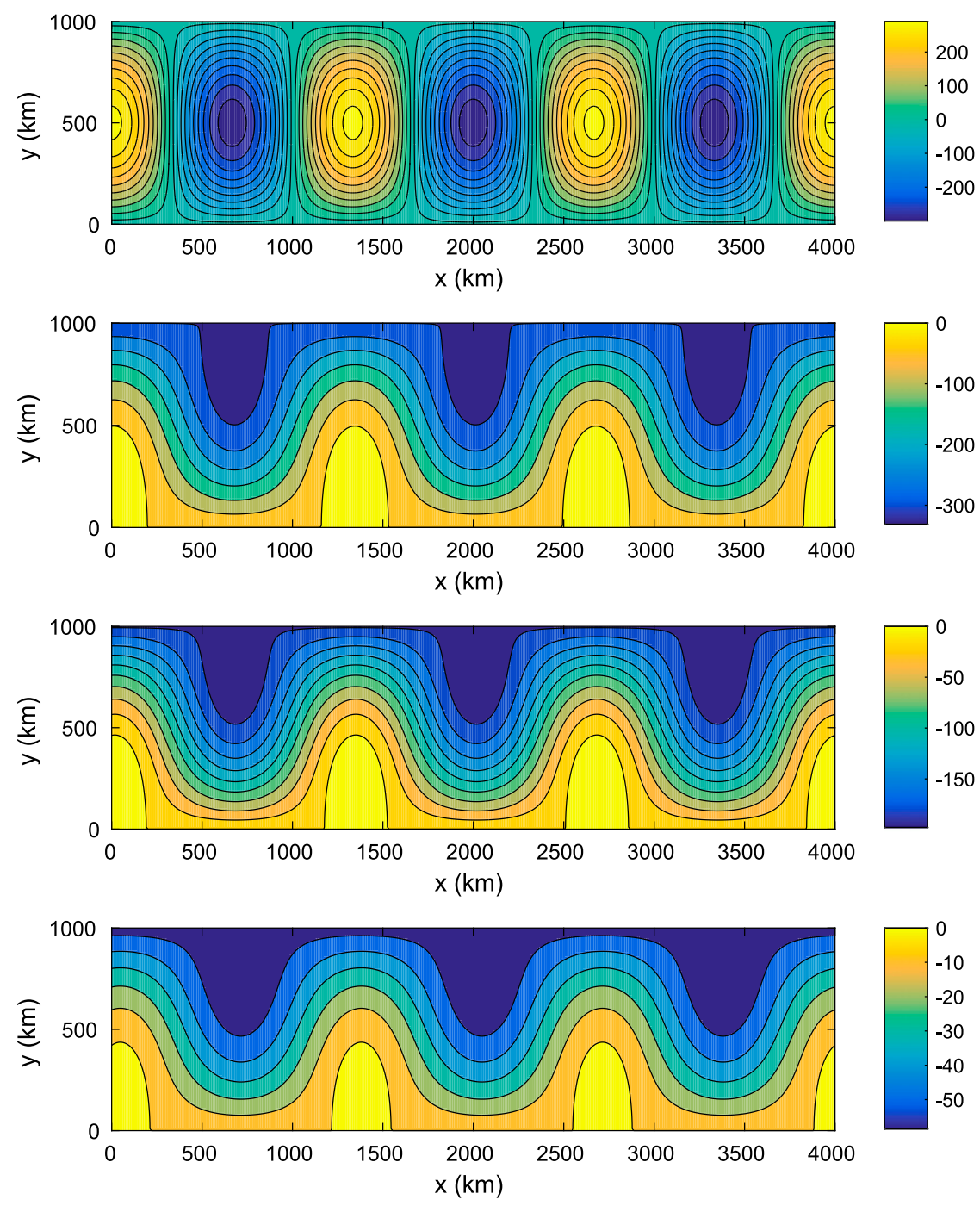

FIG. 2. (top) Bottom topography (m) represented by $c_{3}=300 \mathrm{~m}$ (case 1 ). Here and in subsequent figures the topographic Fourier coefficients whose values are not explicitly stated are set to zero. Streamfunction $\Psi$, times reference depth $H(\mathrm{~Sv})$, with $k_{0}=$ (second row) 0 , (third row) 200, and (fourth row) $400 \mathrm{~m}^{2} \mathrm{~s}^{-1}$.

$E_{U}$ and $E_{V}$ occur when $k_{0}=0$, and kinetic energy decreases with increasing $k_{0}$ (see Figs. 5-7, lower panels). The component $E_{U}$ may be higher than $E_{V}$ (cases 1 and 2) or lower (case 3 ) depending on the details of the bottom topography.

Increasing wind stress leads to increasing zonal transport (see Fig. 9). In case 3 for $k_{0}=0$ a fivefold increase in wind stress amplitude $\tau_{0}=5 \times 10^{-4} \mathrm{~m}^{2} \mathrm{~s}^{-2}$ results in a factor-3 increase in transport from 115.0 to $338.3 \mathrm{~Sv}$. Note, however, that the transport does not increase linearly with increasing wind stress: the sensitivity reduces by a factor of 2 from $\tau_{0}=1 \times 10^{-4} \mathrm{~m}^{2} \mathrm{~s}^{-2}$ to $\tau_{0}=$ $5 \times 10^{-4} \mathrm{~m}^{2} \mathrm{~s}^{-2}$. Note that this reducing sensitivity of the transport for high values of wind stress does not relate to eddy activity (recall we are considering the case $k_{0}=0$ ). Constantinou and Young (2017) and Constantinou (2018) found barotropic eddy saturation, that is, insensitivity of the transport to wind forcing in QG flow in a barotropic configuration. On the other hand Munday et al. (2013) demonstrated eddy saturation in a three-dimensional baroclinic setting using an oceanonly general circulation model. It would be interesting to verify eddy saturation in our model with parameterized eddies. However, the transport strongly depends on the value of $k_{0}$ (Fig. 9). It would take additional effort to find the best-fitting coefficient $k_{0}$ for each wind stress. One approach would be to perform eddy-resolving GCM experiments with given wind stress. Based on 

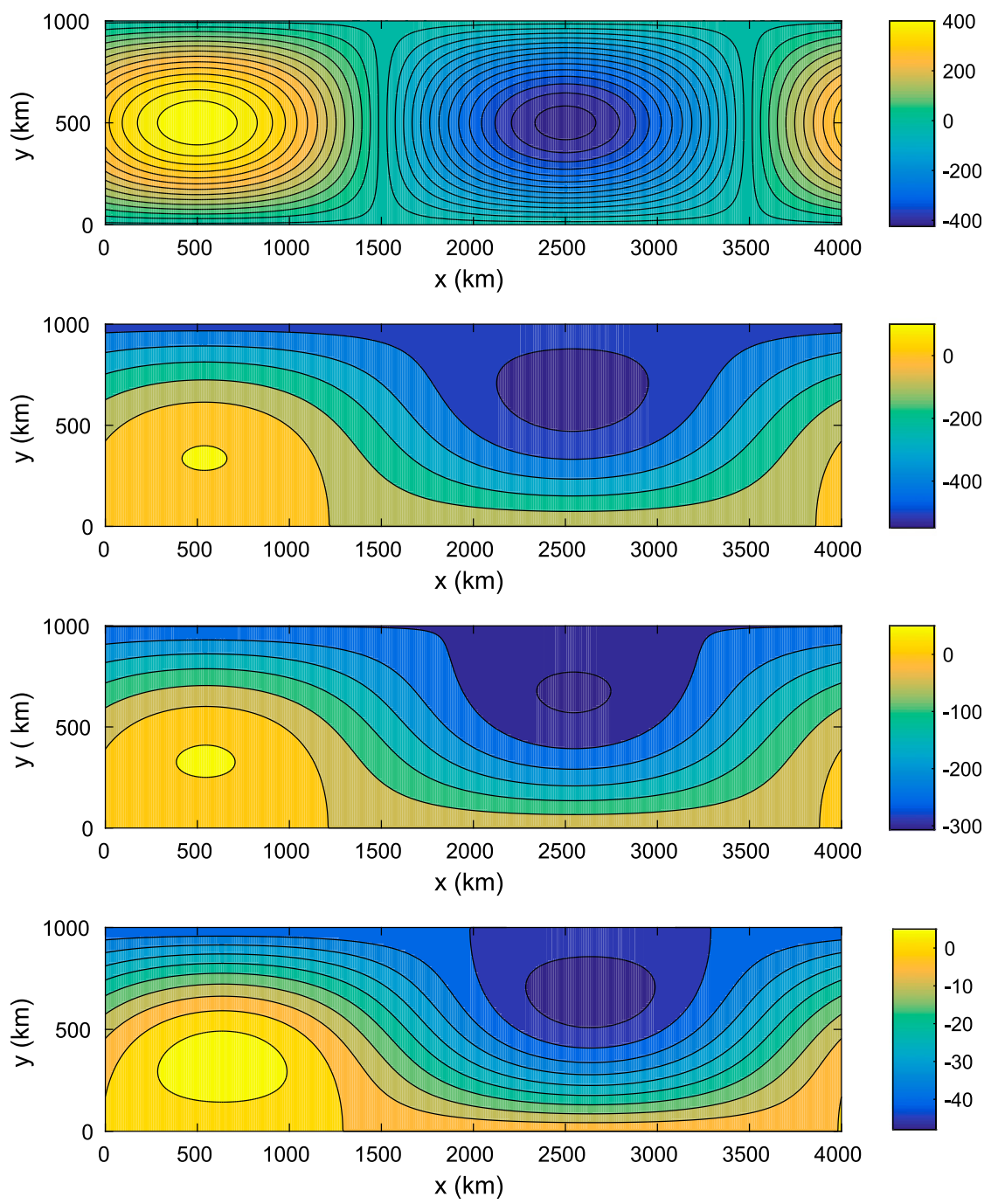

FIG. 3. As in Fig. 2, but for (top) $c_{1}=300 \mathrm{~m}$ and $d_{1}=300 \mathrm{~m}$ (case 2$)$ and $k_{0}=$ (second row) 0 , (third row) 400, and (fourth row) $800 \mathrm{~m}^{2} \mathrm{~s}^{-1}$.

values of transport taken from these eddy-resolving experiments, we could use the relationship between transport and $k_{0}$ (as in Fig. 9) to find the most realistic value of $k_{0}$ for each wind stress and then verify eddy saturation in the parameterized model.

\section{Discussion and conclusions}

Mesoscale eddy parameterization is an important problem of physical oceanography helping to understand the dynamics of interactions of eddies with the mean flow. Moreover, even state-of-the-art highresolution $1 / 12^{\circ}$ global models do not resolve mesoscale eddies in high latitudes.

There are various approaches to the problem of eddy parameterization. This study focuses on parameterization of eddy QGPV fluxes. PV and QGPV are conserved variables, which allows use of a diffusion type of parameterization, contrary to momentum, which is not conserved, and therefore a diffusive parameterization is unsuitable in this case.

Whether the effective coefficient of potential vorticity diffusion is positive represents the principal question in studies of mesoscale eddy parameterization (Welander 1973; Marshall 1981). If the coefficient is of negative sign a diffusive parameterization cannot be used, since it would be both mathematically and physically incorrect. The sign of this coefficient in a zonal barotropic channel is the topic of the present paper. We have demonstrated that if transient eddies are adequately described as effective PV diffusion, then the mean PV diffusivity over the domain $k_{0}$ must 

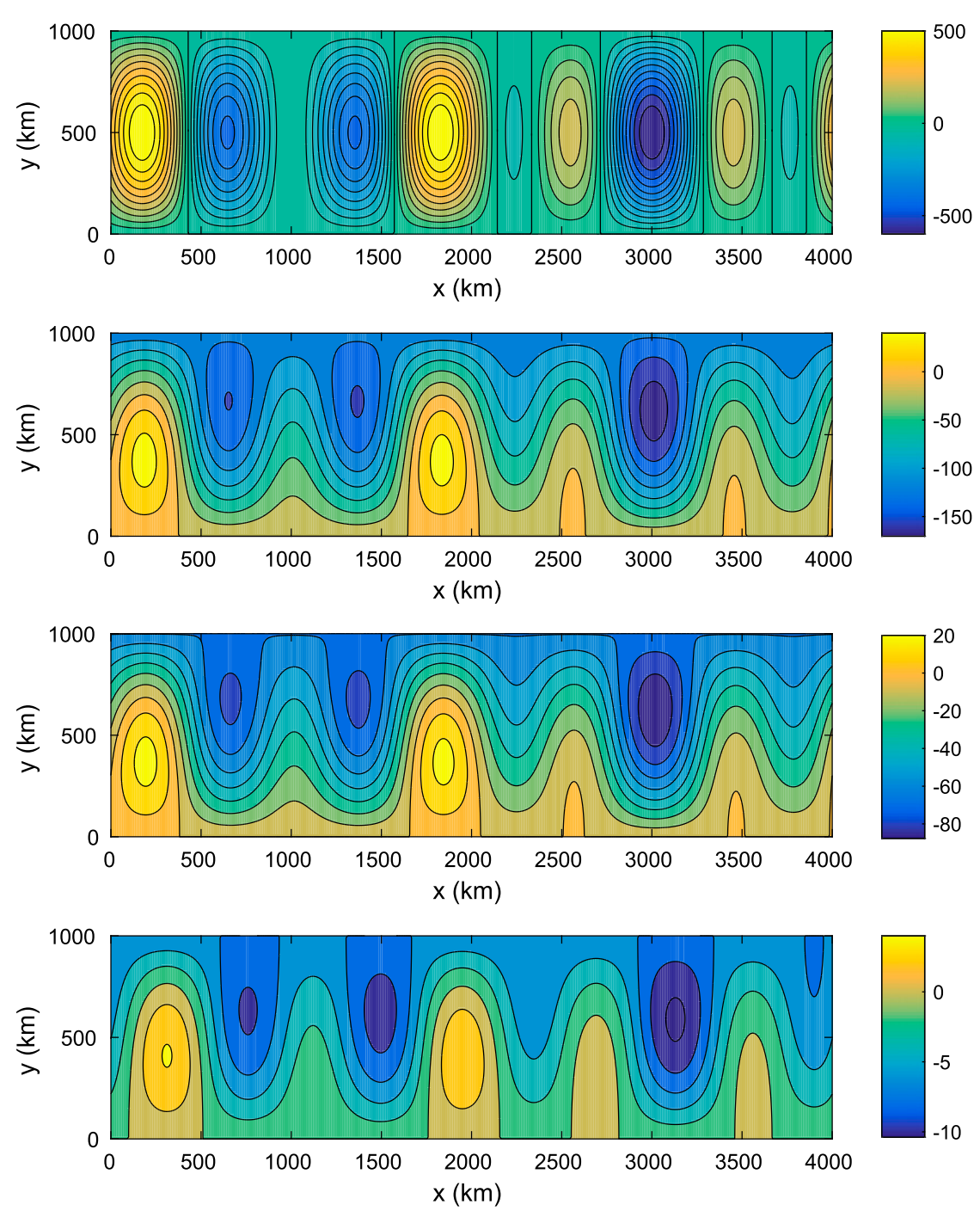

FIG. 4. As in Fig. 2, but for (top) $c_{2}=300 \mathrm{~m}$ and $d_{5}=300 \mathrm{~m}$ (case 3 ) and $k_{0}=$ (second row) 0 , (third row) 100, and (fourth row) $200 \mathrm{~m}^{2} \mathrm{~s}^{-1}$.

be positive in eastward flows. This result comes out of the balance of the zonal momentum and kinetic energy: because of the parameterization, a new term appears in these equations with the physical sense of a topographic form stress for unresolved scales. The main zonal momentum balance is between wind stress [the LHS in Eq. (30)], topographic form stress exerted by the mean flow, and topographic form stress exerted by parameterized eddies.

The integral constraint on meridional fluxes of eddy QGPV known as the theorem of Bretherton in the case of a flat-bottom channel is generalized for barotropic zonal flow under variable-bottom relief. This expression allows us to provide a clear physical sense for the $\beta k$ term, as a topographic form stress exerted by parameterized eddies.
We introduce a new integral measure $D$ of the roughness of the bottom topography, which is the rms of topographic slope. The best-fitting curve representing the relationship between zonal transport and $D$ is of hyperbolic type with a large increase of the transport when $D$ is small and decreasing and a small decrease when $D$ is large and increasing (Fig. 8).

In the kinetic energy balance, the only positive contribution comes from the wind stress $\{E, \tau\}$, which is balanced by eddy diffusion of potential vorticity $\{E, k\}$, eddy diffusion of QGPV linked with topography $\{E, h\}$, bottom dissipation $\{E, \epsilon\}$, and a sink of energy due to topographic form stress by parameterized eddies $\{E, \beta\}$. This correctly explains the mechanism of flow deceleration by eddies associated with nonzero bottom topography, and it 


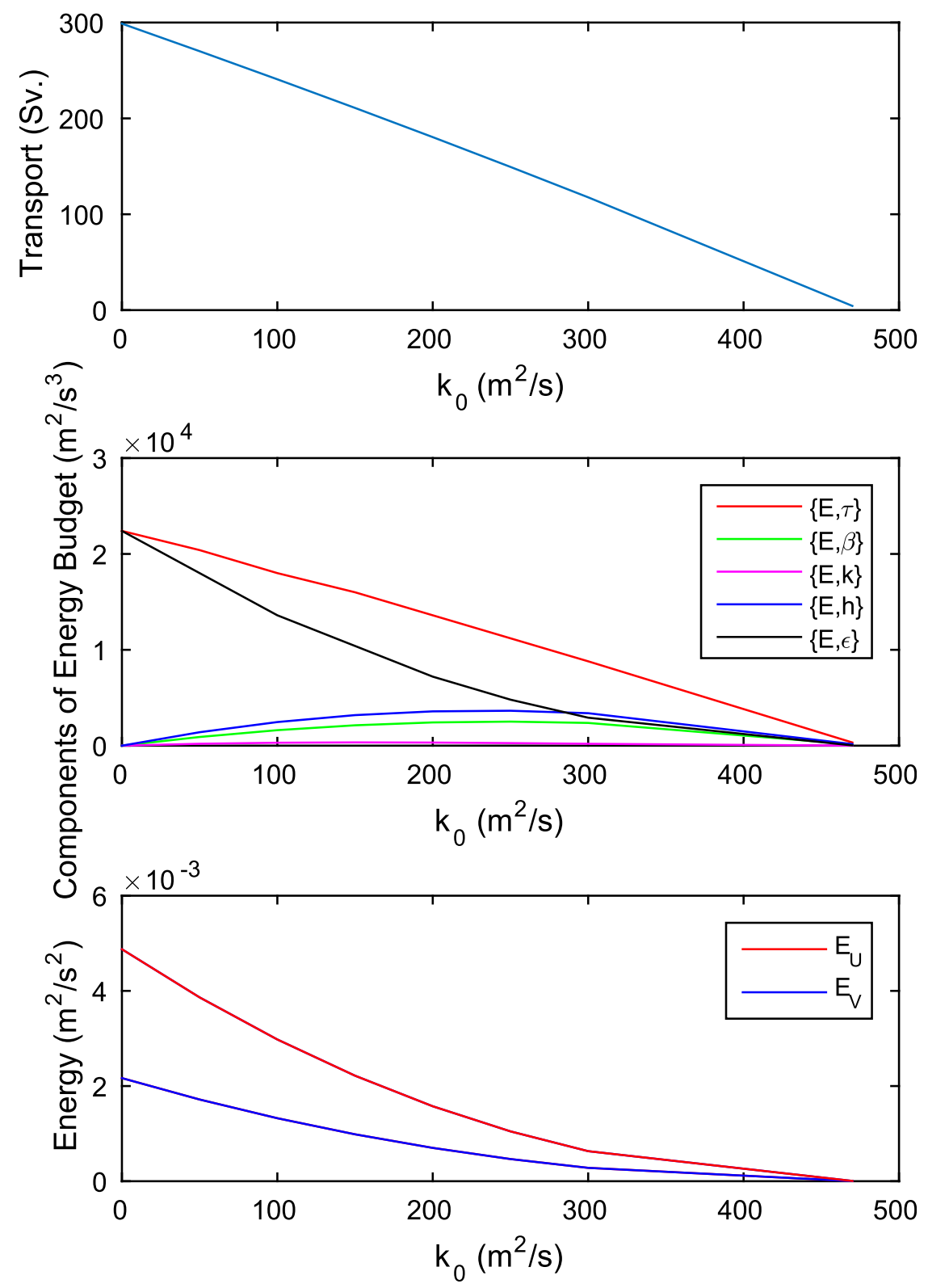

FIG. 5. (top) Zonal transport (Sv) as a function of $k_{0}\left(\mathrm{~m}^{2} \mathrm{~s}^{-1}\right)$. (middle) Components of the domain-averaged energy budget $\{E, \tau\},\{E, \beta\},\{E, k\},\{E, h\}$ and $\{E, \epsilon\}\left(\mathrm{m}^{2} \mathrm{~s}^{-3}\right)$ as functions of $k_{0}\left(\mathrm{~m}^{2} \mathrm{~s}^{-1}\right)$. (bottom) Domain-averaged kinetic energy $E_{U}$ and $E_{V}\left(\mathrm{~m}^{2} \mathrm{~s}^{-2}\right)$ as functions of $k_{0}$. All panels represent case 1 : bottom topography $c_{3}=300 \mathrm{~m}$.

corresponds to downgradient QGPV eddy fluxes in eastward flow. Note that the topographic form stress is the main mechanism balancing the wind stress in the Antarctic Circumpolar Current (Munk and Palmén 1951; McWilliams et al. 1978; Ivchenko et al. 1996; Stevens and Ivchenko 1997; Ivchenko et al. 2008). Provided the diffusion parameterization of eddy PV fluxes holds [i.e., provided Eq. (9) is valid] then the deceleration mechanism of topographic form stress ensures $k_{0}>0$ for eastward (ACC like) flows. Another result of our study is that $k_{0}$ is also constrained to be less than $k_{\max }=\left(\pi \tau_{0}\right) /(4 \beta H)$, because $\{E, \tau\}-\{E, \beta\}$ must be positive [Eq. (51)]. However, for any given choice of the prescribed wind stress and other geometrical and geophysical parameters $k_{0}$ is further constrained (i.e., $\left.k_{0}<k_{\max }^{\text {eff }}<k_{\max }\right)$. In the cases considered we found $k_{\max }<\left(\pi \tau_{0}\right) /(4 \beta H)=1.12 \times 10^{3} \mathrm{~m}^{2} \mathrm{~s}^{-1}$. The individual values of $k_{\max }^{\text {eff }}$ were $4.7 \times 10^{2}, 8.5 \times 10^{2}$, and 

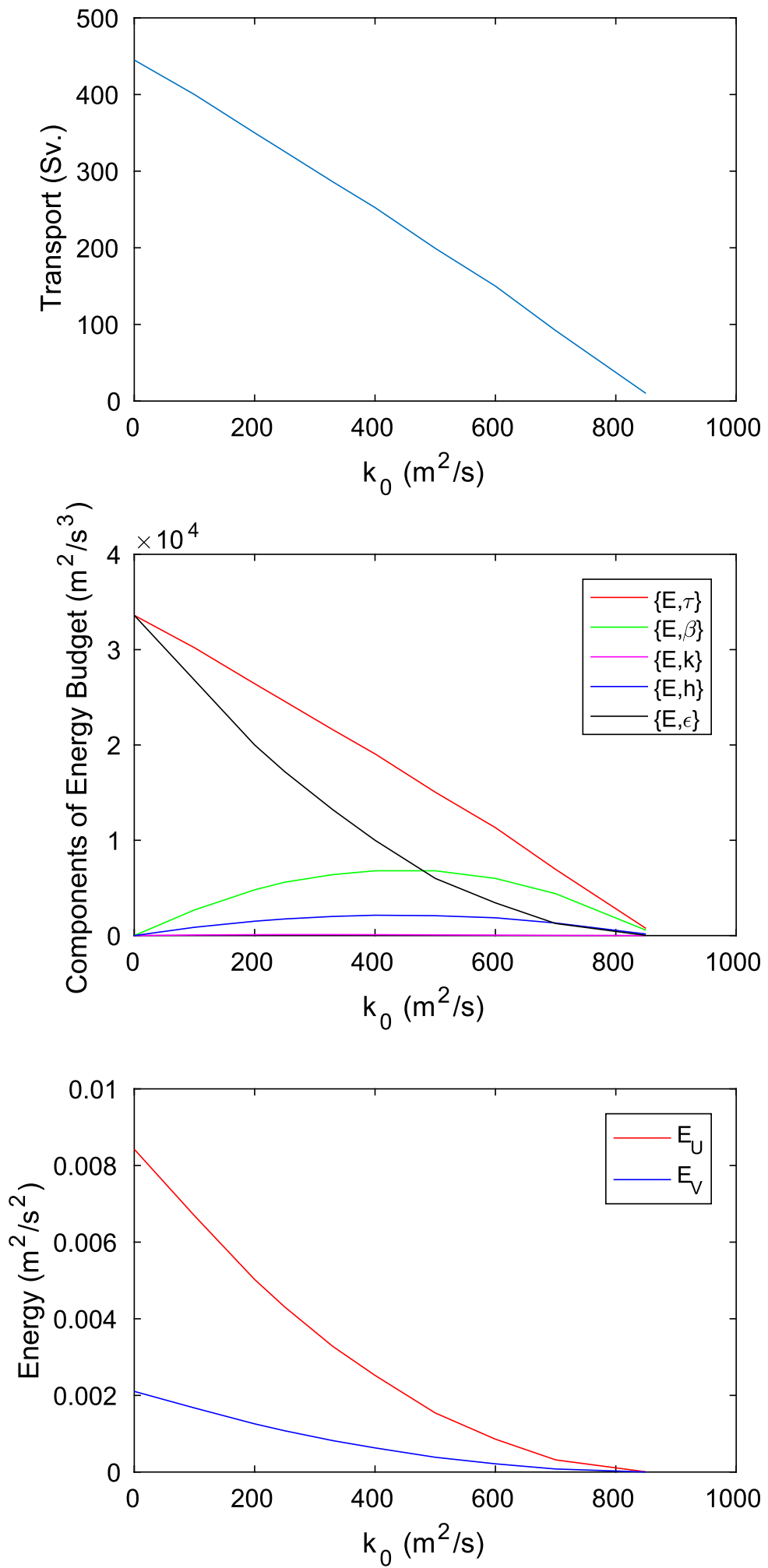

FIG. 6. As in Fig. 5, but for case 2: bottom topography $c_{1}=300 \mathrm{~m}$ and $d_{1}=300 \mathrm{~m}$. 

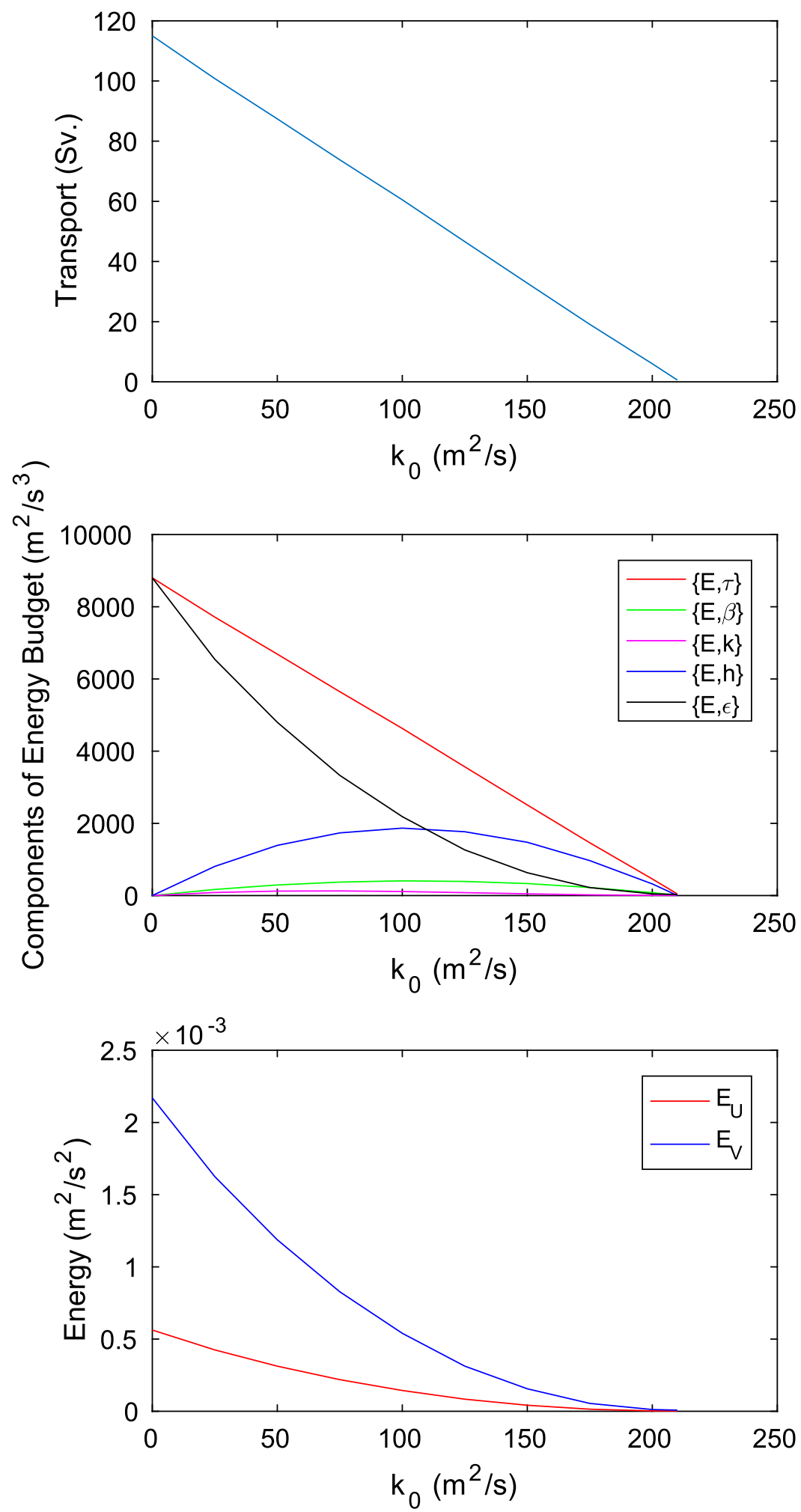

FIG. 7. As in Fig. 5, but for case 3: bottom topography $c_{2}=300 \mathrm{~m}$ and $d_{5}=300 \mathrm{~m}$. 


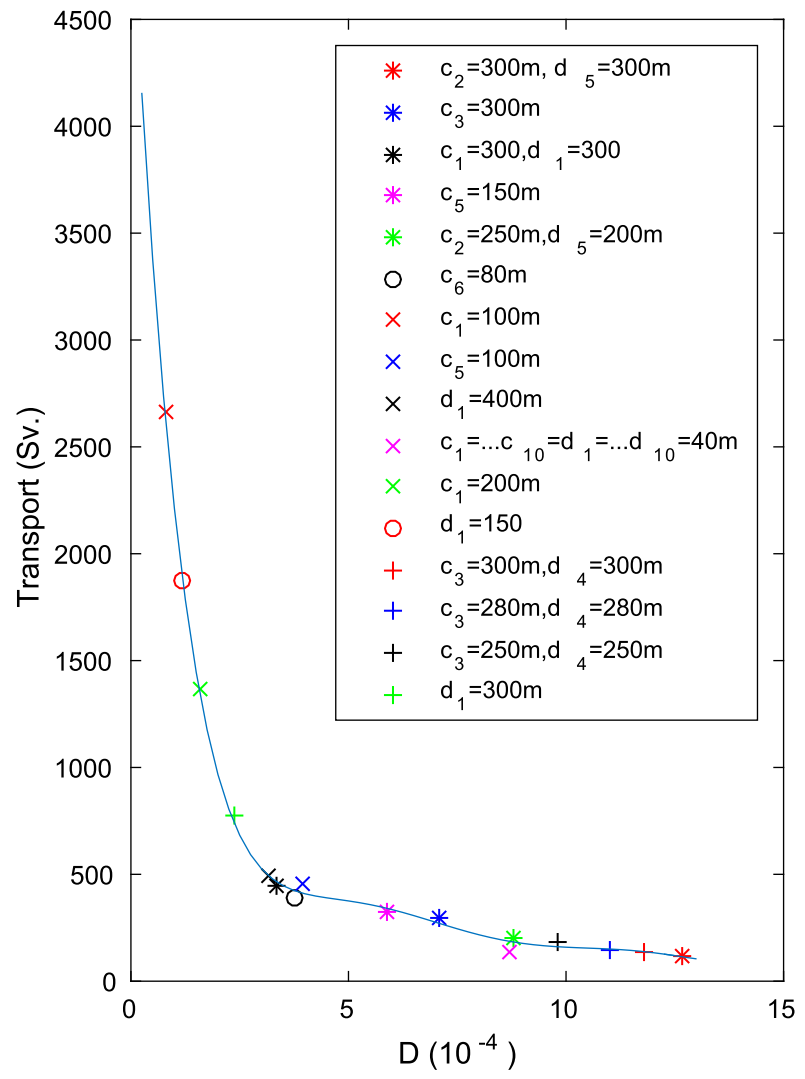

FIG. 8. Scatterplot of zonal transport (Sv) vs parameter of topographic roughness $D$ for various realizations of bottom topography. The fitting curve is based on a seventh-order polynomial approximation.

$2.1 \times 10^{2} \mathrm{~m}^{2} \mathrm{~s}^{-1}$ (for cases 1,2 , and 3 ), respectively (see middle panels of Figs. 5, 6, and 7).

Only modes represented in the bottom topography contribute to the amplitude of the streamfunction $a_{n}$ and $b_{n}$ [see Eqs. (47) and (48)]. Since the modulus of the amplitudes $c_{n}$ and $d_{n}$ of the Fourier topographic modes are finite and $\lim _{n \rightarrow \infty} a_{n}=\lim _{n \rightarrow \infty} b_{n}=0$, one can interpret this as a diminishing contribution of high-frequency modes in topography to mean flow and topographic form stress. This agrees with numerical experiments by Treguier and McWilliams (1990), where they demonstrated that an isolated bottom topography feature of large spatial scale in the path of the ACC generates form stress more efficiently than randomly distributed small-scale topography with the same rms height. They also noted that the domainaveraged topographic form stress is dominated by the contribution from large-scale topography. The authors used a baroclinic QG model; however, it is plausible that a barotropic model would produce qualitatively similar results regarding the influence of bottom

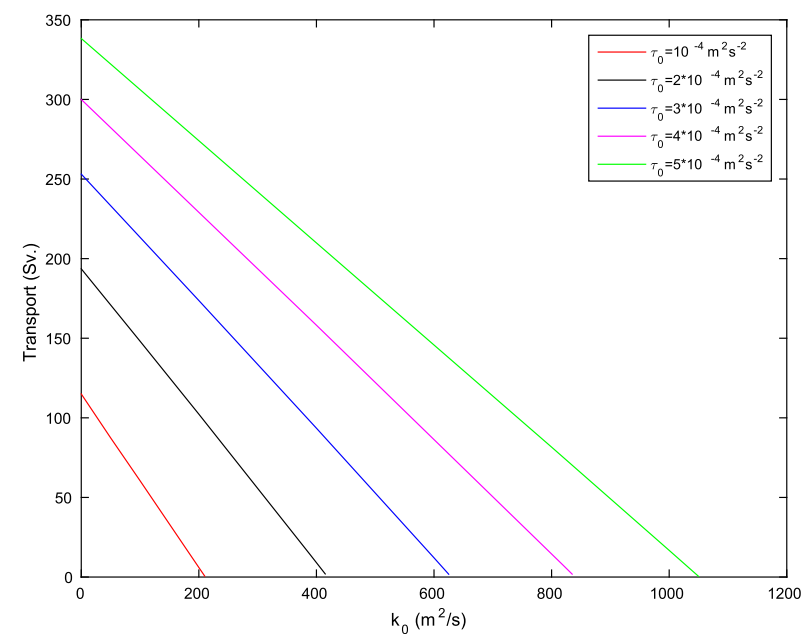

FIG. 9. Zonal transport (Sv) as a function of $k_{0}\left(\mathrm{~m}^{2} \mathrm{~s}^{-1}\right)$ for various wind stress. Case 3: bottom topography $c_{2}=300 \mathrm{~m}$ and $d_{5}=300 \mathrm{~m}$.

topography. Note also that the eddy field in our model would be damped $\left(k_{0} \rightarrow 0\right)$ in the case where $\beta \rightarrow 0$, since $k_{\max } \rightarrow 0$.

Constantinou and Young (2017) demonstrated an "eddy saturation" regime, that is, insensitivity of the zonal transport to large changes in the wind stress (provided the wind stress is over a threshold value), in a barotropic configuration. To study eddy saturation in our model we need to choose a value of $k_{0}$ for each type of topography, since the zonal transport depends strongly on $k_{0}$. In this context, the appropriate $k_{0}$ could be estimated using eddy-resolving GCM experiments. For a given wind stress eddy-resolving model experiments can be used to evaluate the associated transport, and the relationship between transport and $k_{0}$ (similar to Fig. 9) can then be used to obtain an appropriate $k_{0}$. However, this is beyond the scope of the present paper.

In summary, our study demonstrates conclusively that if QGPV diffusion is a good approximation, then the mean QGPV diffusivity must be positive. Our results will contribute to further understanding and parameterization of the effects of mesoscale eddies in more realistic ocean and climate models in the future.

Acknowledgments. We thank two anonymous reviewers for their substantial efforts in reviewing our paper. Their comments were extremely helpful and led to a much improved manuscript. VOI acknowledges the support of the University of Southampton and the National Oceanography Centre. VBZ was 
supported by the Russian Science Foundation, Grant 17-77-30001. BS was supported by National Capability funding from the U.K. Natural Environment Research Council.

\section{APPENDIX}

\section{Parameters and Further Details of the Solution Method}

In developing solutions for $a_{n}$ and $b_{n}$ [Eqs. (47) and (48)] the new parameters introduced are listed here:

$$
\begin{aligned}
R_{0}^{(n)}= & -N^{(n)}-\frac{1}{N^{(n)}} \beta^{2}\left(\frac{2 \pi}{L_{x}}\right)^{2} n^{2}, \\
R_{1}^{(n)}= & \frac{2}{N^{(n)}} M^{(n)} \beta\left(\frac{2 \pi}{L_{x}}\right) n, \\
R_{2}^{(n)}= & -\frac{1}{N^{(n)}} M^{(n) 2}, \\
S_{0}^{(n)}= & -\frac{1}{N^{(n)}} d_{n} k_{0} \frac{f_{0}}{H}\left(\frac{2 \pi}{L_{x}}\right)^{3} n^{3} \beta \\
& -c_{n} k_{0} \frac{f_{0}}{H}\left(\frac{2 \pi}{L_{x}}\right)^{2} n^{2}, \\
S_{1}^{(n)}= & \frac{1}{N^{(n)}} c_{n} \frac{f_{0}}{H}\left(\frac{2 \pi}{L_{x}}\right)^{2} \beta n^{2}+\frac{1}{N^{(n)}} d_{n} k_{0} \frac{f_{0}}{H}\left(\frac{2 \pi}{L_{x}}\right)^{2} M^{(n)} n^{2} \\
& -d_{n} \frac{f_{0}}{H}\left(\frac{2 \pi}{L_{x}}\right)^{n}, \text { and }
\end{aligned}
$$$$
S_{2}^{(n)}=-\frac{1}{N^{(n)}} c \frac{f_{0}}{H} M^{(n)}\left(\frac{2 \pi}{L_{x}}\right) n
$$

Equations (47), (48), and (49) together constitute the desired analytic solution. However, because of the mathematical complexity we are unable to obtain explicit solutions for $U$ in terms of the external parameters. Instead we apply an inverse solution method. We seek values of $U$ that are consistent with the specified wind stress $\tau_{0}$ (e.g., $10^{-4} \mathrm{~m}^{2} / \mathrm{s}^{2}$ in the standard case). We make an initial guess of $U$ and substitute this in Eqs. (47) and (48) to give initial estimates of $a_{n}$ and $b_{n}$. These estimates are then substituted in Eq. (49) to obtain a corresponding value of $\tau_{0}$. In general the initial guess does not yield the desired value of $\tau_{0}$. We therefore increase/decrease the guessed value of $U$ and repeat the procedure until we find the value of $U$ that yields the desired value of $\tau_{0}$ (to within $0.1 \%$ ) (see Fig. A1). To determine the relationship between $k_{0}$ and $U$ for a given wind stress, the above procedure is repeated for a variety of choices of $k_{0}$.

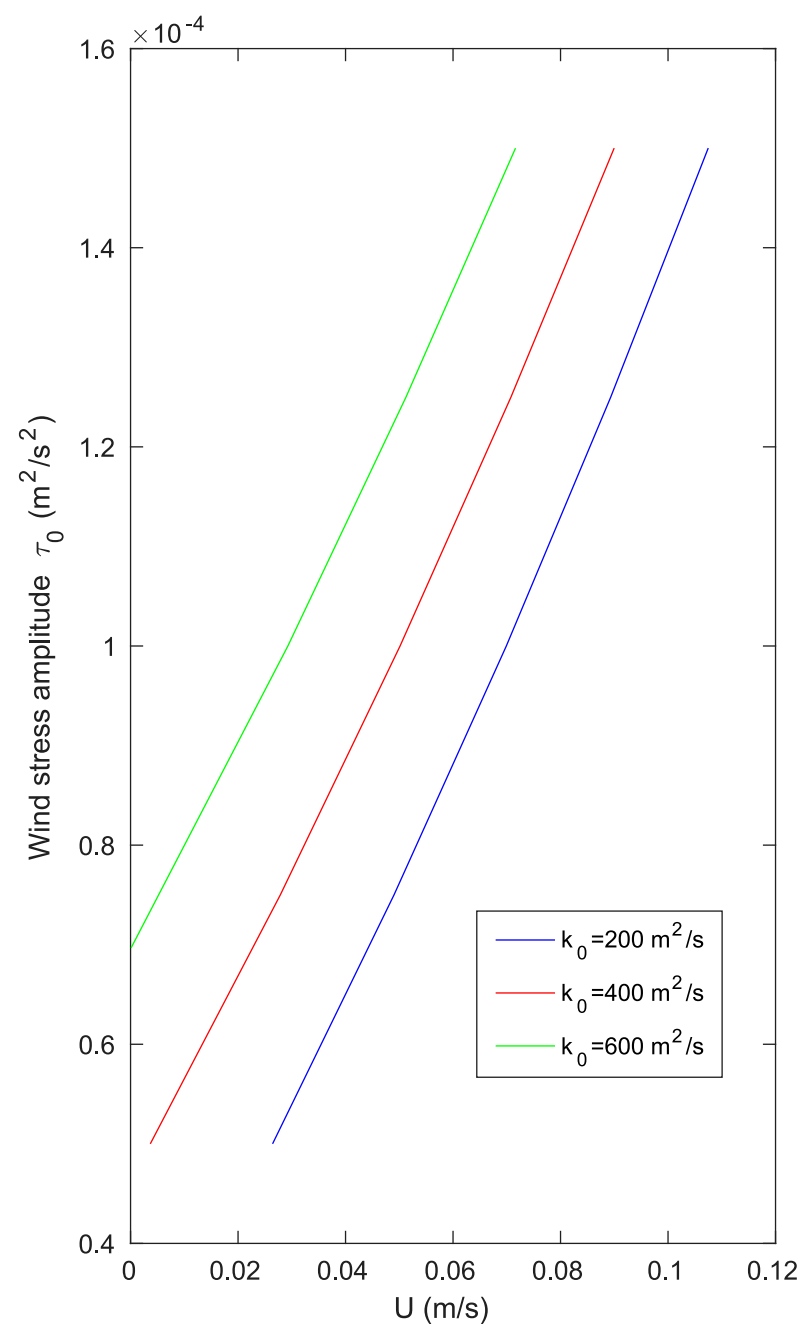

FIG. A1. Relationship between the mean zonal velocity $U$ and the wind stress amplitude $\tau_{0}$. Case 2 : bottom topography $c_{1}=300 \mathrm{~m}$ and $d_{1}=300 \mathrm{~m}$.

\section{REFERENCES}

Abernathey, R., D. Ferreira, and A. Klocker, 2013: Diagnostics of isopycnal mixing in a circumpolar channel. Ocean Modell., 72, 1-16, https://doi.org/10.1016/j.ocemod.2013.07.004.

Bachman, S., and B. Fox-Kemper, 2013: Eddy parameterization challenge suite I: Eady spindown. Ocean Modell., 64, 12-28, https://doi.org/10.1016/j.ocemod.2012.12.003.

Birner, T., D. W. J. Thompson, and T. G. Sheperd, 2013: Upgradient eddy fluxes of potential vorticity near the subtropical jet. Geophys. Res. Lett., 40, 5988-5993, https://doi.org/10.1002/ 2013GL057728.

Bretherton, F. S., 1966: Critical layer instability in baroclinic flows. Quart. J. Roy. Meteor. Soc., 92, 325-334, https://doi.org/ 10.1002/qj.49709239302.

Carnevale, G. F., and J. S. Frederiksen, 1987: Nonlinear stability and statistical mechanics of flow over topography. J. Fluid Mech., 175, 157-181, https://doi.org/10.1017/ S002211208700034X. 
Charney, J. G., J. Shukla, and K. C. Mo, 1981: Comparison of a barotropic blocking theory with observation. J. Atmos. Sci., 38, 762-779, https://doi.org/10.1175/1520-0469(1981)038<0762: $\mathrm{COABBT}>2.0 . \mathrm{CO} ; 2$

Constantinou, N. C., 2018: A barotropic model of eddy saturation. J. Phys. Oceanogr., 48, 397-411, https://doi.org/10.1175/ JPO-D-17-0182.1.

— , and W. R. Young, 2017: Beta-plane turbulence above monoscale topography. J. Fluid Mech., 827, 415-447, https:// doi.org/10.1017/jfm.2017.482.

Dritschel, D. G., and M. E. McIntyre, 2008: Multiple jets as PV staircases: The Philips effect and the resilience of eddytransport barriers. J. Atmos. Sci., 65, 855-874, https://doi.org/ 10.1175/2007JAS2227.1.

Eden, C., 2010: Parameterising meso-scale eddy momentum fluxes based on potential vorticity mixing and a gauge term. Ocean Modell., 32, 58-71, https://doi.org/10.1016/ j.ocemod.2009.10.008.

Fox-Kemper, B., R. Ferrari, and J. Pedlosky, 2003: On the indeterminacy of rotational and divergent eddy fluxes. J. Phys. Oceanogr., 33, 478-483, https://doi.org/10.1175/1520-0485 (2003) $033<0478$ :OTIORA $>2.0 . \mathrm{CO} ; 2$.

Gent, P. R., and J. C. McWilliams, 1990: Isopycnal mixing in ocean circulation models. J. Phys. Oceanogr., 20, 150-155, https:// doi.org/10.1175/1520-0485(1990)020<0150:IMIOCM >2.0.CO;2.

Green, J. S. A., 1970: Transfer properties of the large-scale eddies and the general circulation of the atmosphere. Quart. J. Roy. Meteor. Soc., 96, 157-185, https://doi.org/ 10.1002/qj.49709640802.

Ivchenko, V. O., 1984: Parameterization of the eddy fluxes of the quasi-geostrophic potential vorticity in zonal flows. Dokl. Acad. Nauk USSR, 277, 972-976.

1987: Influence of bottom topography on the eddy transfer coefficient. Izv. Acad. Nauk USSR, Fiz. Atmos. Okeana, 23, 200-208.

-, K. J. Richards, and D. P. Stevens, 1996: The dynamics of the Antarctic Circumpolar Current. J. Phys. Oceanogr., 26, 753-774, https://doi.org/10.1175/1520-0485(1996)026<0753: TDOTAC $>2.0 . \mathrm{CO} ; 2$

,-- B. Sinha, and J.-O. Wolff, 1997: Parameterization of mesoscale eddy fluxes in zonal ocean flows. J. Mar. Res., 55, 1127-1162, https://doi.org/10.1357/0022240973224076.

_ , S. Danilov, and D. Olbers, 2008: Eddies in numerical models of the Southern Ocean. Ocean Modeling in an Eddying Regime, Geophys. Monogr., Vol. 177, Amer. Geophys. Union, 177-198.

, B. Sinha, V. B. Zalesny, R. Marsh, and A. T. Blaker, 2013: Influence of bottom topography on integral constraints in zonal flows with parameterized potential vorticity fluxes. J. Phys. Oceanogr., 43, 311-323, https://doi.org/10.1175/JPO-D-12-0126.1.

— S. Danilov, B. Sinha, and J. Schroeter, 2014a: Integral constraints for momentum and energy in zonal flows with parameterized potential vorticity fluxes: Governing parameters. J. Phys. Oceanogr., 44, 922-943, https://doi.org/10.1175/ JPO-D-13-0173.1.

,-- , and J. Shroeter, 2014b: Comparison of the effect of parameterized eddy fluxes of thickness and potential vorticity. J. Phys. Oceanogr., 44, 2470-2484, https://doi.org/10.1175/ JPO-D-13-0267.1.

Kamenkovich, V. M., M. N. Koshlyakov, and A. S. Monin, 1986: Synoptic Eddies in the Ocean. D. Reidel Publishing Company, 433 pp.

Killworth, P. D., 1997: On the parameterization of eddy transfer: Part I. Theory. J. Mar. Res., 55, 1171-1197, https://doi.org/ 10.1357/0022240973224102.
Maddison, J. R., D. P. Marshall, and J. Shipton, 2015: On the dynamical influence of ocean eddy potential vorticity fluxes. Ocean Modell., 92, 169-182, https://doi.org/10.1016/j.ocemod.2015.06.003.

Mak, J., J. R. Maddison, and D. P. Marshall, 2016: A new gaugeinvariant method for diagnosing eddy diffusivities. Ocean Modell., 104, 252-268, https://doi.org/10.1016/j.ocemod.2016.06.006.

Marshall, D. P., and A. J. Adcroft, 2010: Parameterization of ocean eddies: Potential vorticity mixing, energetics and Arnold's first stability theorem. Ocean Modell., 32, 188-204, https://doi.org/ 10.1016/j.ocemod.2010.02.001.

— J. R. Maddison, and P. S. Berloff, 2012: A framework for parameterizing eddy potential vorticity fluxes. J. Phys. Oceanogr., 42, 539-557, https://doi.org/10.1175/JPO-D-11-048.1.

Marshall, J. C., 1981: On the parameterization of geostrophic eddies in the ocean. J. Phys. Oceanogr., 11, 257-271, https:// doi.org/10.1175/1520-0485(1981)011<0257:OTPOGE>2.0.CO;2.

McWilliams, J. C., 2008: The nature and consequences of oceanic eddies. Ocean Modeling in an Eddying Regime, Geophys. Monogr., Vol. 177, Amer. Geophys. Union, 5-15.

— A reference solution in a $\beta$-plane channel. J. Phys. Oceanogr., 11, 921-949, https://doi.org/10.1175/1520-0485(1981)011<0921: EGTIAR $>2.0 . \mathrm{CO} ; 2$.

— W. R. Holland, and J. S. Chow, 1978: A description of numerical Antarctic circumpolar currents. Dyn. Atmos. Oceans, 2, 213-291, https://doi.org/10.1016/0377-0265(78)90018-0.

Munday, D. R., H. L. Johnson, and D. P. Marshall, 2013: Eddy saturation of equilibrated circumpolar currents. J. Phys. Oceanogr., 43, 507-532, https://doi.org/10.1175/JPO-D-12-095.1.

Munk, W. H., and E. Palmén, 1951: Note on the dynamics of the Antarctic Circumpolar Current. Tellus, 3, 53-55, https:// doi.org/10.3402/tellusa.v3i1.8609.

Olbers, D., 2005: On the role of eddy mixing in the transport of zonal ocean currents. Marine Turbulence: Theories, Observations, and Models, H. Baumert, J. Simpson, and J. Sündermann, Eds., Cambridge University Press, $630 \mathrm{pp}$.

_ J. Wolff, and C. Voelker, 2000: Eddy fluxes and second-order moment balances for nonhomogeneous quasigeostrophic turbulence in wind-driven zonal flows. J. Phys. Oceanogr., 30, 1645-1668, https://doi.org/10.1175/1520-0485(2000)030<1645: EFASOM $>2.0 . \mathrm{CO} ; 2$.

Rhines, P. B., and W. R. Young, 1982: Homogenization of potential vorticity in planetary gyres. J. Fluid Mech., 122, 347-367, https://doi.org/10.1017/S0022112082002250.

Ringler, T., and P. Gent, 2011: An eddy closure for potential vorticity. Ocean Modell., 39, 125-134, https://doi.org/10.1016/ j.ocemod.2011.02.003.

Sinha, B., 1993: The influence of mesoscale eddies and topography on Southern Ocean flow. Ph.D. thesis, Southampton University, $197 \mathrm{pp}$.

Smith, D. M., S. Cusack, A. W. Colman, C. K. Folland, G. R. Harris, and J. M. Murphy, 2007: Improved surface temperature prediction for the coming decade from a global climate model. Science, 317, 796-799, https://doi.org/10.1126/science.1139540.

Stevens, D. P., and V. O. Ivchenko, 1997: The zonal momentum balance in an eddy-resolving general-circulation model of the Southern Ocean. Quart. J. Roy. Meteor. Soc., 123, 929-951, https://doi.org/10.1002/qj.49712354008.

Treguier, A. M., and J. C. McWilliams, 1990: Topographic influences on wind-driven, stratified flow in a $\beta$-plane channel An idealized model for the Antarctic Circumpolar Current J. Phys. Oceanogr., 20, 321-343, https://doi.org/10.1175/ 1520-0485(1990)020<0321:TIOWDS>2.0.CO;2. 
- I. M. Held, and V. D. Larichev, 1997: Parameterization of quasigeostrophic eddies in primitive equation ocean models. J. Phys. Oceanogr., 27, 567-580, https://doi.org/10.1175/15200485(1997)027<0567:POQEIP > 2.0.CO;2.

Vallis, G. K., 2006: Atmospheric and Oceanic Fluid Dynamics: Fundamentals and Large-Scale Circulation. Cambridge University Press, 745 pp.

Wardle, R., and J. Marshall, 2000: Representation of eddies in primitive equation models by a PV flux. J. Phys. Oceanogr., 30, 2481-2503, https://doi.org/10.1175/1520-0485(2000)030<2481: ROEIPE $>2.0 . \mathrm{CO} ; 2$.

Welander, P., 1973: Lateral friction in the ocean as an effect of potential vorticity mixing. Geophys. Fluid Dyn., 5, 173-189, https://doi.org/10.1080/03091927308236114.
Wolff, J.-O., E. Maier-Reimer, and D. J. Olbers, 1991: Wind-driven flow over topography in a zonal $\beta$-plane channel: A quasigeostrophic model of the Antarctic Circumpolar Current. J. Phys. Oceanogr., 21, 236-264, https://doi.org/10.1175/15200485(1991)021<0236:WDFOTI >2.0.CO;2.

Wood, R. B., and M. E. McIntyre, 2010: A general theorem on angular-momentum changes due to potential vorticity mixing and on potential-energy changes due to buoyancy mixing. J. Atmos. Sci., 67, 1261-1274, https://doi.org/10.1175/ 2009JAS3293.1.

Wunsch, C., and D. Stammer, 1995: The global frequencywavenumber spectrum of oceanic variability estimated from TOPEX/POSEIDON altimetric measurements. J. Geophys. Res., 100, 24 895-24 910, https://doi.org/10.1029/95JC01783. 